\title{
Aberration and coherence effects with a micromirror array
}

\section{Benton, David}

David Benton, "Aberration and coherence effects with a micromirror array," Proc. SPIE 11867, Technologies for Optical Countermeasures XVIII and HighPower Lasers: Technology and Systems, Platforms, Effects V, 118670D (12 September 2021); doi: 10.1117/12.2597501

SPIE. Event: SPIE Security + Defence, 2021, Online Only 


\title{
Aberration and Coherence Effects with a Micromirror Array
}

\author{
David Benton \\ Aston Institute of Photonic Technologies, Aston University, Birmingham, UK. B4 7ET \\ d.benton@aston.ac.uk
}

\begin{abstract}
Micro mirror arrays, such as the Digital Micromirror Device (DMD) from Texas Instruments, are amplitude modulating spatial light modulators. Binary holograms write spatial amplitude modulations to the device and with coherent sources can be used to control reflected beams through controlled diffraction. In this paper the inherent aberrations within the DMD device have been identified and corrected through the use of Zernike modes written to the array allowing a device profile to be used as a foundation characteristic upon which other applications can be improved. The approach used coopts a technique used for wavelength measurement using a DMD which focusses onto a camera. The tilting action of the mirrors means the device acts as a mixture of a lens and 2D blazed grating. In this case it also allows assessments of coherence length to be made based on a dual dispersion capability. A model for producing the Fresnel diffraction pattern from a specific DMD pattern is produced using Fresnel diffraction theory and shows good agreement with measured results.
\end{abstract}

\section{INTRODUCTION}

The Digital Micromirror Device (DMD) produced by Texas Instruments is an addressable array of tilting micromirrors which acts as a spatial light modulator (SLM). The micromirrors tilt at angles of $+12^{\circ}$ and $-12^{\circ}$ redirecting the incident light in 2 directions and, when coherent light is used, acting as a binary hologram. This binary amplitude SLM is then well suited for use with binary diffractive optics patterns such as the Fresnel zone plate(FZP) and has been used as a method of beam steering [1][2]. The concept behind the FZP is that the binary amplitude function is used to remove light that would contribute to the focus amplitudes with one polarity (i.e. negative amplitudes) ensuring that all remaining amplitudes then contribute positively to the resultant intensity. The concept of super pixels has been used to select a more specific phase from within a group of pixels (say $4 \times 4$ ) which is precise but less efficient than polarity selection [3]. The principle of polarity selection can be applied more widely than to focussing. In this work I describe the use of Zernike modes on a DMD to produce controlled wavefronts. The intention is to use a DMD to emulate atmospheric scintillation effects by imposing aberration patterns upon a reflected wavefront [4], taking advantage of the high frame rate available with DMDs in comparison to liquid crystal SLMs. However the particular focus here in this work is in overcoming the inherent aberrations of the device itself, atmospheric emulation will be published separately and will build upon this work. This is done by improving the focussing properties of the DMD device using a camera and can be used as a device specific profile. The use of a FZP upon a DMD has previously been used in order to assess incident wavelength [5] but made no account for device aberrations. The DMD behaves as both a blazed diffraction grating and a focussing element which has implications for the point spread function. A theory is presented for calculating the expected Fresnel diffraction pattern from a given DMD pattern. The work here is presented in 2 sections, the first of which (Wavefront construction) looks at writing Zernike modes to a DMD and measuring the effects upon a focus. The second section concentrates on modelling the expected diffraction results from a DMD pattern.

\section{WAVEFRONT CONSTRUCTION.}

The shape of a wavefront can be represented by a series of orthogonal Zernike modes which correspond to traditional Seidal aberrations of tip/tilt, defocus, astigmatism, coma etc. The Zernike modes are usually represented in terms of the radial and azimuthal position of a point on a surface, but with a 2D array of squares it makes sense to use a Cartesian form and the first 15 terms are shown in Table 1.

Technologies for Optical Countermeasures XVIII and High-Power Lasers: Technology and Systems, Platforms, Effects V, edited by D. H. Titterton, R. J. Grasso, M. A. Richardson, W. L. Bohn, H. Ackermann, Proc. of SPIE Vol. 11867, 118670D · C 2021 SPIE · CCC code: 0277-786X/21/\$21 · doi: 10.1117/12.2597501 


\begin{tabular}{|c|c|c|c|}
\hline $\mathbf{j}$ & Zernike term & Cartesian Form & Name \\
\hline 0 & $\mathrm{Z}_{0}$ & 1 & Piston \\
\hline 1 & $\mathrm{Z}_{1}$ & $\mathrm{x}$ & Tip \\
\hline 2 & $\mathrm{Z}_{2}$ & $\mathrm{y}$ & Tilt \\
\hline 3 & $\mathrm{Z}_{3}$ & $2 x y$ & Astigmatism \\
\hline 4 & $\mathrm{Z}_{4}$ & $-1+2 x^{2}+2 y^{2}$ & Defocus \\
\hline 5 & $\mathrm{Z}_{5}$ & $-x^{2}+y^{2}$ & Astigmatism \\
\hline 6 & $\mathrm{Z}_{6}$ & $-x^{3}+3 x y^{2}$ & Trefoil \\
\hline 7 & $\mathrm{Z}_{7}$ & $-2 x+3 x^{3}+3 x y^{2}$ & Coma \\
\hline 8 & $\mathrm{Z}_{8}$ & $-2 y+3 y^{3}+3 x^{2} y$ & Coma \\
\hline 9 & $\mathrm{Z}_{9}$ & $y^{3}-3 x^{2} y$ & Trefoil \\
\hline 10 & $\mathrm{Z}_{10}$ & $-4 x^{3} y+4 x y^{3}$ & Quadrafoil \\
\hline 11 & $\mathrm{Z}_{11}$ & $-6 x y+8 x^{3} y+8 x y^{3}$ & Secondary astigmatism \\
\hline 12 & $Z_{12}$ & $1-6 x^{2}-6 y^{2}+6 x^{4}+6 y^{4}+12 x^{2} y^{2}$ & Spherical aberration \\
\hline 13 & $\mathrm{Z}_{13}$ & $3 x^{2}-3 y^{2}-4 x^{4}+4 y^{4}$ & Secondary astigmatism \\
\hline 14 & $Z_{14}$ & $x^{4}-6 x^{2} y^{2}+y^{4}$ & Quadrafoil \\
\hline
\end{tabular}

Table 1 Cartesian form Zernike mode terms

The distorted wavefront at the DMD plane $W\left(x_{0}, y_{0}\right)$ is formed from the sum of all Zernike modes

$$
W\left(x_{0}, y_{0}\right)=\sum a_{j} Z_{j}
$$

Where the factor $a_{j}$ is a mode dependent amplitude and the values $\mathrm{x}_{0}$ and $\mathrm{y}_{0}$ are relative to an origin in the centre of the mirror array. Once the wavefront is composed and converted to a wavelength $(\lambda)$ dependent phase value, a binary pixel value $\mathrm{D}\left(\mathrm{x}_{0}, \mathrm{y}_{0}\right)$ is selected:

$$
\begin{aligned}
& \mathrm{D}\left(\mathrm{x}_{0}, \mathrm{y}_{0}\right)=0 \text { if } \sin \left[\frac{2 \pi}{\lambda} W\left(\mathrm{x}_{0}, \mathrm{y}_{0}\right)\right]<0 \\
& \mathrm{D}\left(\mathrm{x}_{0}, \mathrm{y}_{0}\right)=1 \text { if } \sin \left[\frac{2 \pi}{\lambda} W\left(\mathrm{x}_{0}, \mathrm{y}_{0}\right)\right] \geq 0
\end{aligned}
$$

The Zernike modes are only orthogonal for a circular aperture and hence an opaque circle must be written with a radius of the smallest dimension of the array.

The DMD aberrations are exposed by examining the focal quality from a simple system involving collimated light, the DMD and a camera. The system is shown schematically in Figure 1. In an ideal system a FZP written to the DMD would produce a diffractive focus onto a camera, with the size of the FZP chosen appropriately for the DMD to camera distance.

The DMD and control electronics used was obtained from Vialux, a DLP 650L with a 1280x800 array of mirrors with $10.8 \mu \mathrm{m}$ spacing. Focus spots were captured with a Thorlabs DCC1545M monochrome camera. A bespoke LabView program was written to calculate Zernike modes, write them to the DMD and capture the peak focus values from the camera. A low cost laser diode at 630nm was used to illuminate the DMD.

Because the DMD reflects head-on incident light at an angle of $24^{\circ}$, there is significant astigmatism present. The FZP can be entirely represented by the defocus $\left(Z_{4}\right.$ term) thus the amplitude $a_{4}$ controls the focal length and the astigmatism is corrected using the $a_{3}$ and a5 values. The focus quality can be determined by measuring the width of the focussed spot on the camera and adjusting to produce the narrowest [7]. When the aberration is not too severe this can be effectively done by simply maximising the peak intensity value. Care must be taken initially when setting the focus parameter as the effects of astigmatism will peak the intensity away from the true focus. This is then set manually before any corrections are made by selecting the focussed spot to be circular (the circle of least confusion) and falling between the horizontal and vertical line focus produced by astigmatism. Each parameter can then be scanned to look for the highest intensity 
and set accordingly. A selection the of the scanned values of Zernike mode amplitudes is shown in Figure 2 where the peak intensity on the camera is plotted against mode amplitude. In each case the amplitude value giving the maximum intensity was chosen. A combined plot of optimum Zernike mode values and the peak intensity achieved with those values is presented in Figure 3. As expected, the major contributions are from focus and astigmatism but higher order modes then produce a $100 \%$ improvement beyond them. The focus and astigmatism terms are a result of the off-axis focus geometry, the higher order terms then represent the inherent aberrations of the DMD itself.

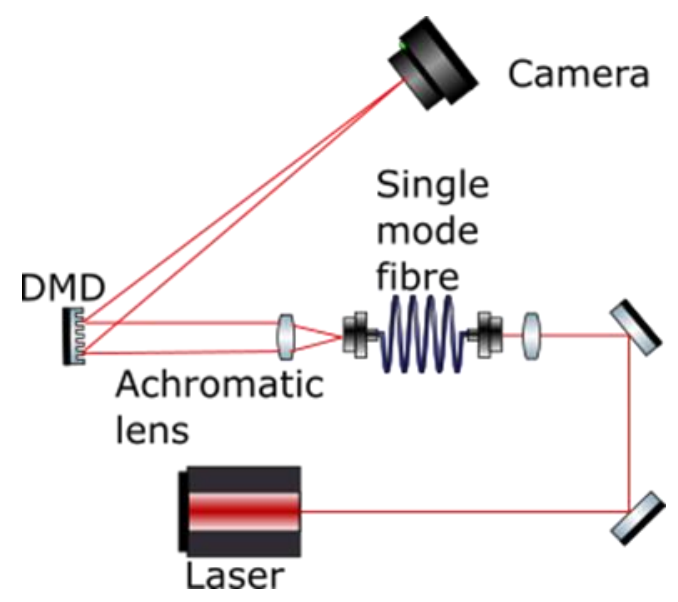

Figure 1. A schematic diagram of the set up for wavefront control measurement of a DMD

These inherent aberration terms represent the device specific profile for the operating wavelength and should scale with other wavelengths. When calculating DMD patterns for other applications, such as beam steering or atmospheric emulation for example, these device profiles should be the base upon which other applications are built and will improve performance and accuracy in those applications. 


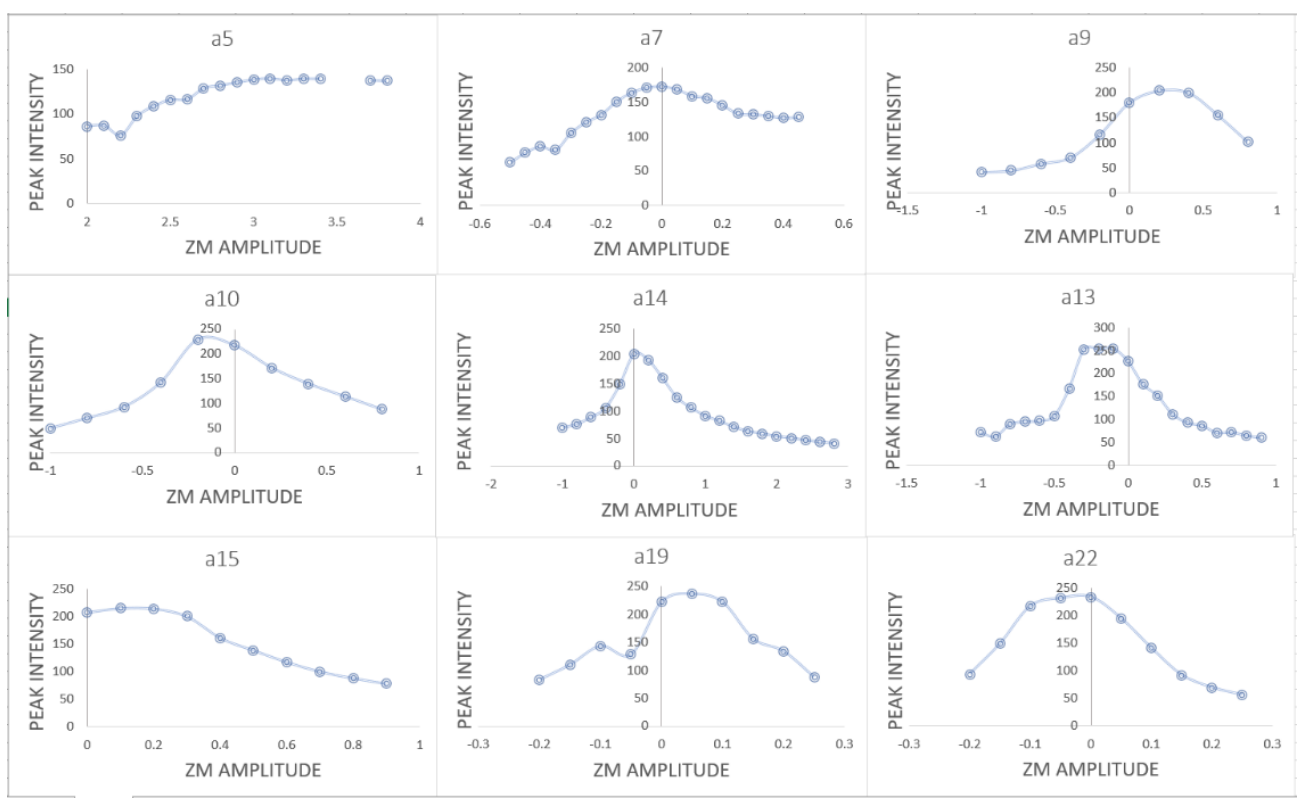

Figure 2 A selection of Zernike mode amplitudes showing the variation in peak intensity as they are adjusted

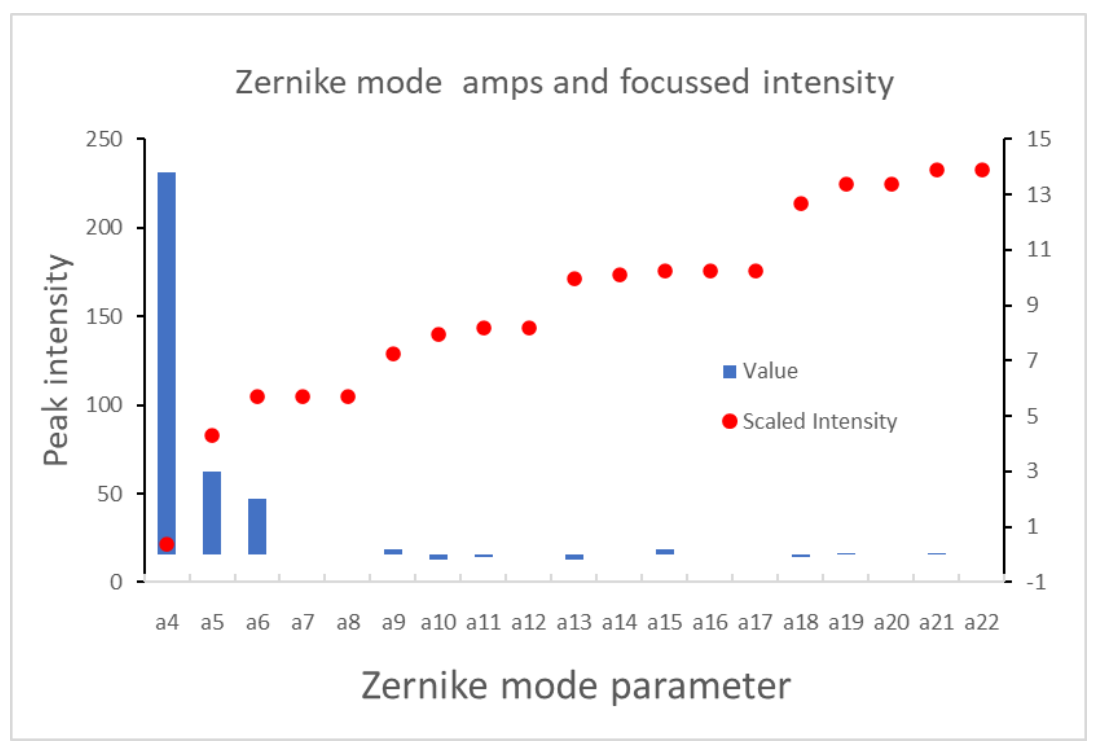

Figure 3. Zernike mode amplitude values for optimum focus and peak intensities after the amplitude is applied.

\section{MODELLING DMD DIFFRACTION PATTERNS}

The approach used here to model the Fresnel diffraction pattern follows closely that presented by Roger Easton [8]. The complex amplitude at some point in the image plane $U(x, y)$, due to a source with amplitude distribution $A\left(x_{0}, y_{0}\right)$ is given by the Rayleigh Sommerfeld equation 


$$
U(x, y)=\frac{1}{i \lambda} \iint A\left(x_{0}, y_{0}\right) \frac{e^{i \frac{2 \pi}{\lambda} R}}{R} d R
$$

Where $\lambda$ is the wavelength of the light being used, and $R$ is the distance from source to image plane point $(x, y)$.

The field at a point in the image plane $(x, y, z)$ arising from a point in the origin source plane $\left(x_{0}, y_{0}, 0\right)$

$$
E\left(x, y, z ; x_{0}, y_{0}, 0\right)=\frac{E_{0}}{i z \lambda} \operatorname{Re}\left(e^{i 2 \pi\left(\frac{z}{\lambda}-v t\right)} e^{\frac{i \pi}{z \lambda}\left[\left(x-x_{0}\right)^{2}+\left(y-y_{0}\right)^{2}\right]}\right)
$$

With $\mathrm{E}_{0}$ the source amplitude, and $v$ the frequency of the light field. The initial multiplying term represents the inverse square reduction with distance. The first exponential term is a phase factor related to the distance travelled. The second exponential term is a phase dependence for off axis positions in source and image planes.

We write the exponential phase dependent terms as a function

$$
h(x, y ; z, \lambda)=\frac{1}{i z \lambda} e^{i 2 \pi\left(\frac{z}{\lambda}-v t\right)} e^{i \pi\left[\left(\frac{x}{\sqrt{z \lambda}}\right)^{2}+\left(\frac{y}{\sqrt{z \lambda}}\right)^{2}\right]}
$$

The Fresnel diffraction pattern is the convolution of the amplitude distribution function $f\left(x_{0}, y_{0}\right)$ with this function $h(x, y)$

$$
\begin{gathered}
g(x, y: z)=\iint f\left(x_{0}, y_{0}\right) h\left(x-x_{0}, y-y_{0} ; z, \lambda\right) d x_{0} d y_{0} \\
=f\left(x_{0}, y_{0}\right) * h(x, y ; z \lambda)
\end{gathered}
$$

By writing $\alpha=\sqrt{z \lambda}$ in the function $h(x, y)$

$$
h(x, y ; z, \lambda)=\frac{1}{i \alpha^{2}} e^{i 2 \pi\left(\frac{z}{\lambda}-v t\right)} e^{i \pi\left[\left(\frac{x}{\alpha}\right)^{2}+\left(\frac{y}{\alpha}\right)^{2}\right]}
$$

we can see that $\alpha$ is the characteristic scale of this function, which represents a quadratic phase variation with respect to the axis, $\alpha$ is the off axis radial distance at which the phase changes by $\pi$. Plotting the phase of this function shows a Gabor zone plate, and $\alpha$ is the chirp rate. The function $h(x, y)$ is more recognisable as the point spread function (PSF) and can be seen in Figure 4. 


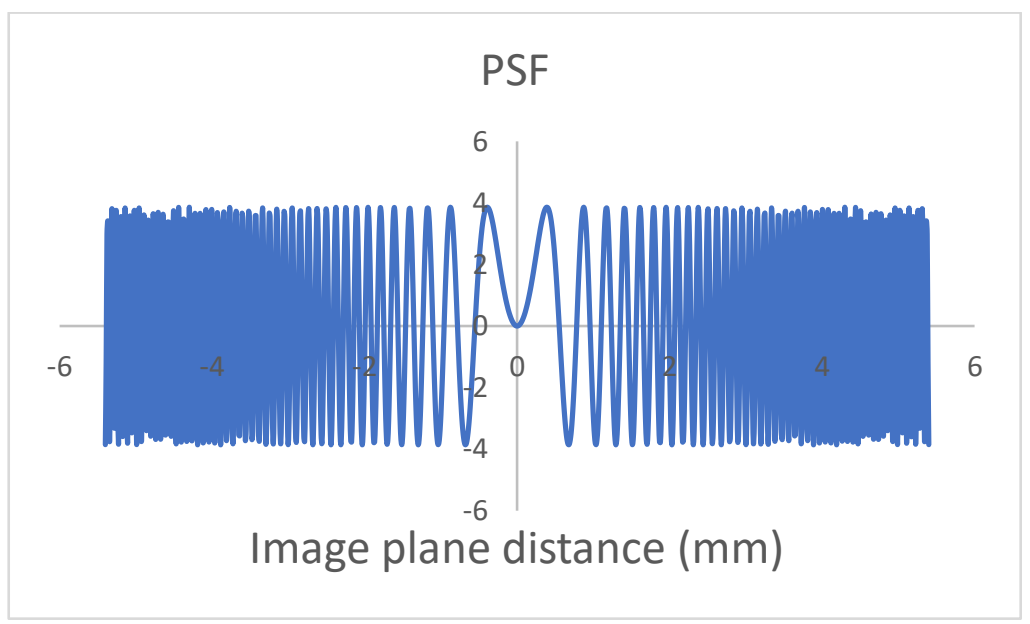

Figure 4. The calculated point spread function at a distance of $50 \mathrm{~cm}$ from the DMD. This is a central slice through the $2 D \mathrm{DSF}$

The intensity distribution in the image plane is the multiplication of the convolution by its complex conjugate.

$$
I(x, y, z)=g(x, y: z) \overline{g(x, y ; z)}
$$

For a given distance $\mathrm{z}$ we need to calculate the PSF only once and hence the calculation of the intensity profile can be quickly computed from the convolution, using the convolution theorem with the Fourier Transform of the PSF and the source function.

The DMD is a $2 \mathrm{D}$ grating and grating orders are generated according to

$$
\mathrm{n}=\sin \varphi \frac{\lambda}{d}
$$

Where $\varphi$ is the angle of the order relative to the axis (assuming input is on axis), $n$ is the order number, and $d$ is the spacing of the DMD mirrors $=10.8 \mu \mathrm{m}$. The mirrors tilt at $12^{\circ}$ to the normal and hence the angle orders appear around the $24^{\circ}$ direction. For $\lambda=630 \mathrm{~nm}$ the order number is 7 . Changing wavelength causes a change in the order angle which results in a movement of the centre of the diffraction pattern. The calculated PSF is centred in the image space so we need to offset the PSF to observe dispersion effects. The change in order angle is effectively linear with wavelength and movement in the image plane is given by

$$
\Delta x=\Delta \varphi \cdot z=\frac{n\left(\lambda-\lambda_{r}\right) z}{d}
$$

Where $\lambda_{\mathrm{r}}$ is a reference wavelength and $\Delta x$ is the translation in the image plane relative to the position of $\lambda_{\mathrm{r}}$. For sources which are non-monochromatic each wavelength within the spectral profile gives rise to a PSF which is shifted relative to the centre wavelength, and each PSF adds incoherently. Thus for the DMD the resultant PSF is a convolution of the monochromatic PSF with the spectral distribution. 

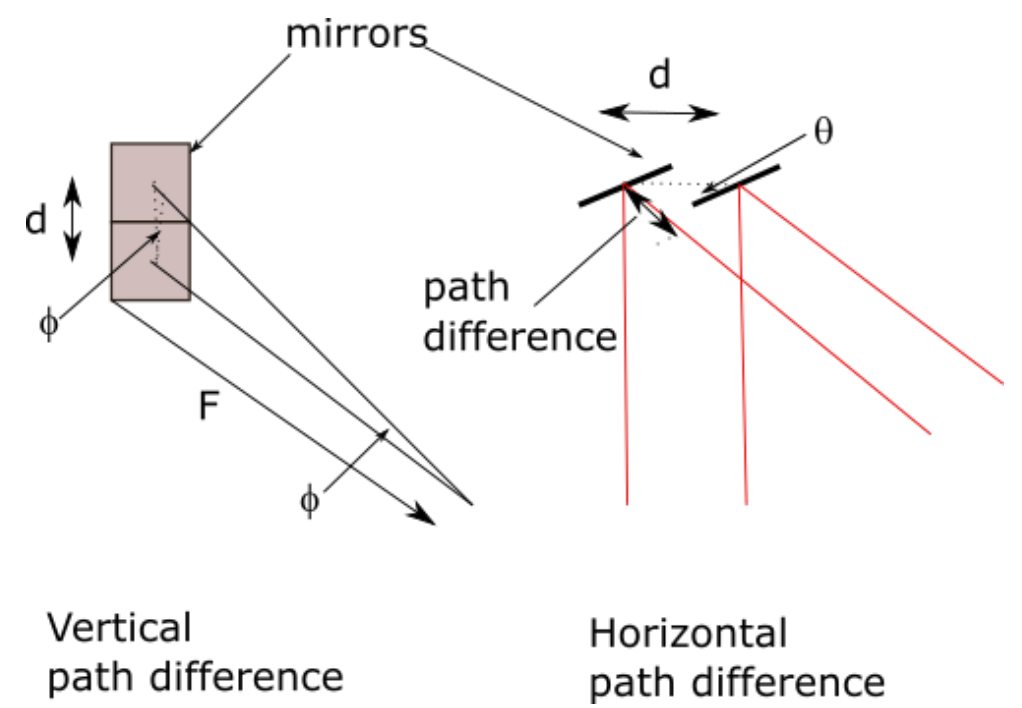

\section{Horizontal path difference}

Figure 5. Diagrams representing the optical path difference between light from neighbouring mirrors

With a conventional FZP we would expect an increase in the spectral bandwidth to increase the focal spot radius - like chromatic aberration. However the DMD is behaving as both a FZP and a blazed grating - a blazed FZP. For mirrors that tilt in the, say, horizontal plane the path length difference between vertically neighbouring mirrors $p_{v}$ (see Figure 5) is given

$$
p_{v}=d \cdot \sin (\Phi)
$$

Where $d$ is the mirror spacing $(10.8 \mu \mathrm{m})$ and $\Phi$ is the small angle subtended to the focal point at distance $\mathrm{F}$

$$
\tan \Phi \approx \Phi=\frac{d}{F}
$$

In the horizontal plane the path difference $p_{h}$ is

$$
p_{h}=2 d \sin (\theta)
$$

Where $\theta$ is the mirror tilt angle, in this case $12^{\circ}$. For a typical lab scale focal length $\mathrm{F}=50 \mathrm{~cm}$, the ratio of horizontal to vertical path difference is to a good approximation

$$
\frac{p_{h}}{p_{v}}=\frac{2 F \sin (\theta)}{d} \approx 19,250
$$

This has implications for the coherence length of the illumination source as for short coherent length sources coherence can be maintained across the entire array in a vertical sense but only for a limited number of mirrors in the horizontal direction. Thus for a spectrally broad source the diffraction focus can be well defined in the non-tilting direction whilst being poorly defined and extended in the tilting (dispersing direction). The blazed FZP then gives us 2 scales of coherence length interaction in the same device which is an interesting addition to the toolset for laser beam detection and characterisation, where coherence length is a valuable diagnostic measurement. 


\section{RESULTS}

Figure 6 shows the results of modelling the intensity distributions from a FZP with the DMD dimensions and an intended focal length of $31 \mathrm{~cm}$. The binary DMD image is multiplied by a 2D Gaussian intensity distribution function representing the radial intensity variation of an illuminating laser source. The distance from DMD to image plane is varied and the results clearly show a sharp focus at the intended focal length of the FZP.

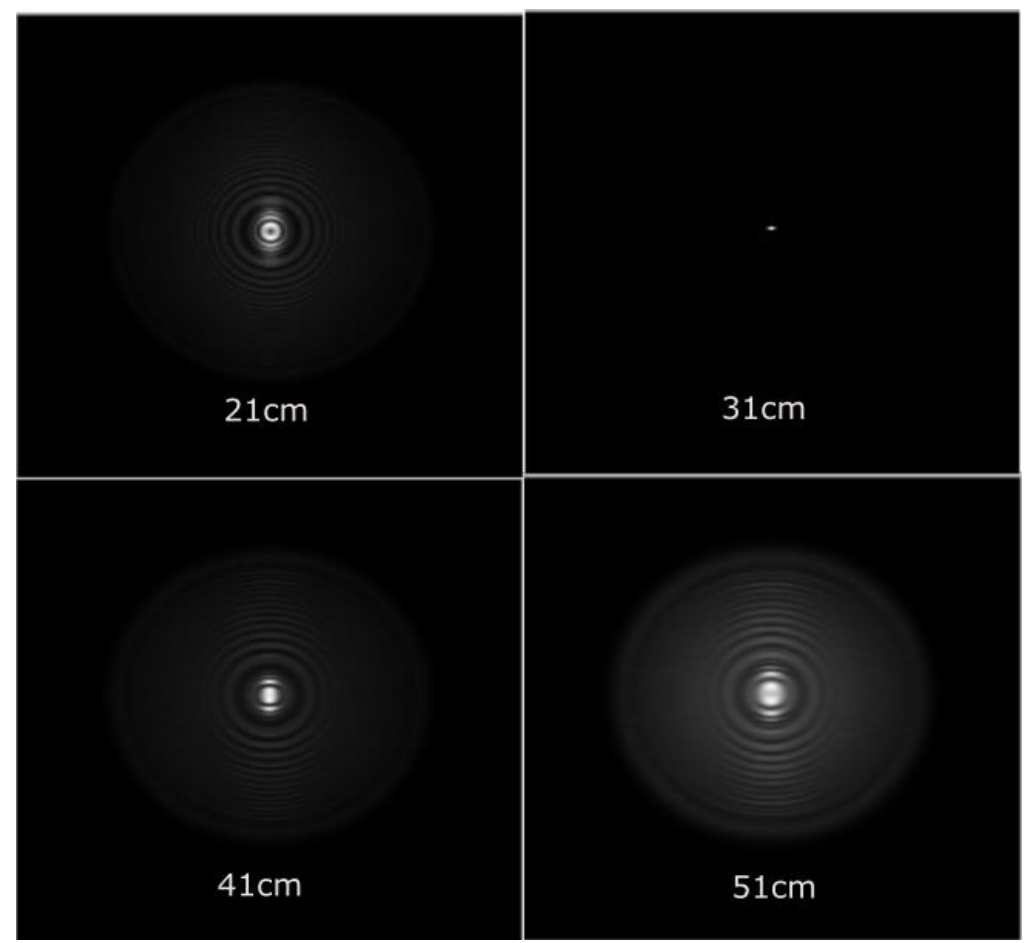

Figure 6. Modelling results of the intensity, normalised to peak value, in image planes at the given separation from DMD. These are from a FZP designed to focus at $31 \mathrm{~cm}$.

The effect of increasing source spectral bandwidth is shown in Figure 7 where the imaging plane is chosen to be at the intended focal length of the FZP. The upper plots are for the focal spot intensity distribution and the lower plots represent the PSF resulting from the spectral bandwidth, with spectral bandwidths of $0.1 \mathrm{~nm}, 1 \mathrm{~nm}$ and $10 \mathrm{~nm}$. The dispersion in the blaze direction shows clearly as the focal spot extends. This was observed experimentally using an LED. A $617 \mathrm{~nm}$ LED (Thorlabs M617F2) with a spectral bandwidth of $12 \mathrm{~nm}$ coupled to a multimode fibre was butted to a single mode fibre and collimated before striking the DMD. A camera at the FZP focal plane recorded a line focus as can be seen in Figure 8, showing good agreement with the model presented above. The value of defocus (a4) was adjusted in the FZP generating function to ensure the camera was seeing the best focus. The FZP generating function included Zernike mode corrections for the inherent aberrations of the DMD and astigmatism - as identified at close-by wavelength of $632 \mathrm{~nm}$. 
$0.1 \mathrm{~nm}$

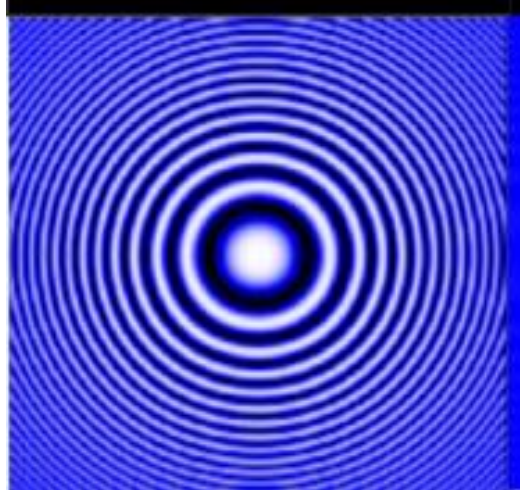

$1 \mathrm{~nm}$

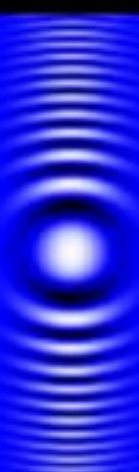

\section{$10 \mathrm{~nm}$}

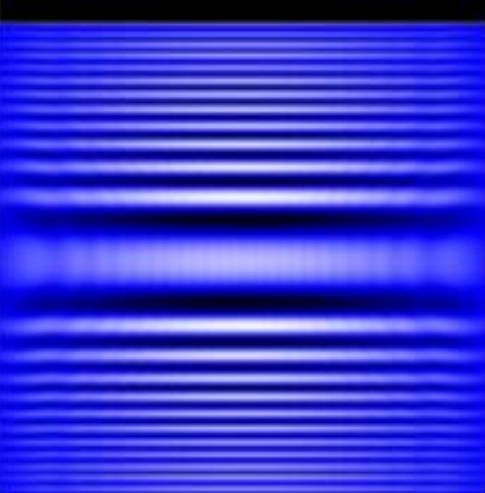

Figure 7 Focus spot intensity distributions (top plots) and corresponding point spread functions (lower plots) for source spectral widths of $0.1 \mathrm{~nm}$ (left), $1 \mathrm{~nm}$ (centre) and 10nm (right)

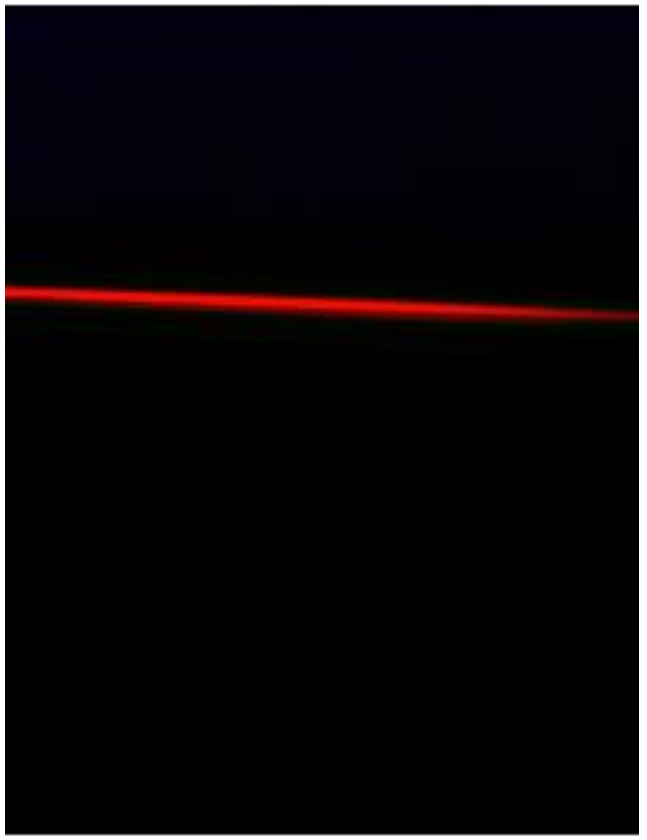

Figure 8. Image captured on camera of extended focal spot from an LED at $617 \mathrm{~nm}$

Proc. of SPIE Vol. 11867 118670D-9

Downloaded From: https://www.spiedigitallibrary.org/conference-proceedings-of-spie on 22 Nov 2021 Terms of Use: https://www.spiedigitallibrary.org/terms-of-use 


\section{DISCUSSION}

The DMD as a spatial light modulator in conjunction with Zernike modes is a useful tool for controlling wavefronts, but has rarely been used to characterise the aberrations of the DMD itself. I have presented here a process for characterising the DMD aberrations such that a device profile can be generated which can be used in conjunction with additional Zernike mode adjustments and wavefront manipulations thereby improving image quality or the accuracy of wavefront representation. The accurate and rapid modelling of the DMD diffraction pattern importantly is made with the ideal pattern and thus assumes that the profile will be subtracted from the ideal pattern before the DMD pattern is implemented.

The observation of a differential coherence response in orthogonal DMD directions is both relevant and potentially useful. Consider that a source with wavelength $630 \mathrm{~nm}$ and a spectral width of $1 \mathrm{~nm}$ has a coherence length of $0.26 \mathrm{~mm}$. A diffraction order of 7 therefore has a path difference of $4.4 \mu \mathrm{m}$ between each pixel and thus the coherence length is exceeded after 60 pixels - which could be referred to as the coherent pixel number. This can be observed in the central PSF of Figure 7 where the PSF is washed out in the horizontal direction. DMD pixels beyond this extent are essentially not contributing to the diffraction pattern (other than adding a constant background). The LED as used in Figure 8 has a coherent pixel number of 5 in the tilt direction. In the orthogonal direction the coherent pixel number exceeds the size of the DMD array. Thus from an efficiency point of view the effective size of the DMD array is reduced if the coherent pixel number is less than the array dimension. This will have implications for the resolution that can be achieved which is proportional to the number of contributing mirror pixels, and is reducing the effective aperture size. This could also be viewed as placing a limit on the minimum focal length that should be written to the DMD to obtain best resolution and efficiency for a given spectral bandwidth.

Determination of coherence length is a valuable characteristic of a laser detector and a device with differential coherence response such as this DMD can render 2 distinct interaction scales which could be utilised. Multiplying the horizontal path difference from equation 13 by the number of mirrors in the horizontal direction gives a total path difference across the DMD of $4.6 \mathrm{~mm}$, which will be the minimum source coherence length to avoid loss of coherence.

\section{Acknowledgements}

The author acknowledges the support of EPSRC grant number EP/T009012/1. The author would like to thank Marie Zandi for supplying the camera image of the focussed LED light.

\section{REFERENCES:}

[1] Benton, D. M. (2017). Multiple beam steering using dynamic zone plates on a micro-mirror array. Optical Engineering, 57(7), 1-13. https://doi.org/10.1117/1.OE.57.7.073109

[2] Lindle, J. R., \& Watnik, A. T. (2018). Large angle nonmechanical laser beam steering at $4.6 \mu \mathrm{m}$ using a digital micromirror device. Optical Engineering, 57(02), 1. https://doi.org/10.1117/1.OE.57.2.027108

[3] Goorden, S. A., Bertolotti, J., \& Mosk, A. P. (2014). Superpixel-based spatial amplitude and phase modulation using a digital micromirror device. Optics Express, 22(15), 17999. https://doi.org/10.1364/oe.22.017999

[4] Anzuola, E., \& Belmonte, A. (2016). Generation of atmospheric wavefronts using binary micromirror arrays. Applied Optics, 55(11), 3039. https://doi.org/10.1364/ao.55.003039

[5] Mohagheghian, M., \& Sabouri, S. G. (2018). Laser wavelength measurement based on a digital micromirror device. IEEE Photonics Technology Letters, 30(13), 1186-1189. https://doi.org/10.1109/LPT.2018.2838262 
[6] Scholes, S., Rodríguez-fajardo, V., Scholes, S., Kara, R., Pinnell, J., Rodríguez-fajardo, V., \& Forbes, A. (2019). Structured light with digital micromirror devices : a guide to best practice, 59(4). https://doi.org/10.1117/1

[7] Benton, D.M, Sugden, K, and Zandi, M "Coherence imaging for laser detection", Proc. SPIE 11537, ElectroOptical and Infrared Systems: Technology and Applications XVII, 115370K (20 September 2020); https://doi.org/10.1117/12.2573194

[8] https://www.cis.rit.edu/class/simg733/Optics_notes_2012-05_small.pdf, Chapter 3. 